www.jmscr.igmpublication.org

Impact Factor 5.84

Index Copernicus Value: 71.58

ISSN (e)-2347-176x ISSN (p) 2455-0450

crossref DOI:_https://dx.doi.org/10.18535/jmscr/v5i11.141

Journal Of Medical Science And Clinical Research

\title{
Incidence of Ankle Arthritis in Syndesmotic Injuries Even after Operative Intervention
}

\author{
Authors \\ Nithyakumar.V.R $\mathbf{R}^{1}$, R.Neelakrishnan ${ }^{2}$ \\ ${ }^{* 1}$ Postgraduate in Orthopaedics, Rajah Muthiah Medical College \& Hospital, Annamalai University \\ ${ }^{2}$ Professor \& HOD of Orthopaedics, Rajah Muthiah Medical College \& Hospital, Annamalai University \\ Email:dr.nithy.2k2@gmail.com
}

\begin{abstract}
Introduction: In a predicted 1-11 percent of sprained ankles, distal tibiofibular syndesmosis got injured. 40 percent of patients have ankle instability even after six months. This is because of widening of the ankle mortise due to stretch of syndesmosis after acute sprain. In fractures of the ankle, syndesmotic damage takes place in approximately 50 percent of type $B$ Weber and in all of type $C$ Weber fractures.

Aim: A Study to assess the incidence of ankle arthritis in syndesmotic disruption even after operative intervention.

Materials \& Methods: All cases of ankle injuries were assessed for syndesmotic disruption and finally taken up for surgery and Follow up achieved at regular time period for durations of 1 year. During the long term follow up few patients encounter ankle arthritis.

Results: Twenty patients with disruption of syndesmotic ligament in ankle injuries had been treated with stainless steel syndesmotic screws and subsequently removed in 8-12 weeks then allowed for weight bearing have studied from june 2015 to September 2017. Few patients had persistent pain in the ankle after weight bearing and ankle arthritis was encountered. The reason for persistent ankle pain and subsequent ankle arthritis was analysed.

Conclusion: It is necessary to fix the syndesmotic disruption with screws or endobutton to prevent ankle arthritis. But even after surgical management there are few patients had persistent ankle pain and subsequent arthritis.

Keywords- syndesmotic disruption, syndesmotic screws, ankle arthritis.
\end{abstract}

\section{Introduction}

Syndesmosis is described as a fibrous joint wherein adjacent bones are related by means of a strong ligaments. This also applies for the tibiofibular syndesmosis of ankle, which is a syndesmotic joint composed of two bones and four ligaments. The distal fibula and tibia share the bony segments and are joined via the distal anterior and posterior tibiofibular ligament, transverse ligament and interosseous ligament. Although syndesmosis is a joint, within the literature the term syndesmotic injury shows injury of the syndesmotic ligaments.

In an estimated 1-11 percent of all ankle sprains, injury of the distal tibiofibular syndesmosis takes place. 40 percent of patients still complaining of ankle instability six months after sprain in the ankle. This might be because of widening of the 
ankle mortise as a result of expanded size of the syndesmotic ligaments after ankle sprain. As widening of the ankle mortise through one $\mathrm{mm}$ increases the space of the tibiotalar joint by 42 $\%^{(1)}$, this will cause instability and therefore early arthritis of the tibiotalar joint.

Syndesmotic disruption can occur in ankle injuries, with or without a fracture. In ankle fractures, syndesmotic get injured in about 50percent of Weber type B and in all Weber type $\mathrm{C}$ fractures, whereas in ankle sprains without fracture, syndesmotic disruption accounts for 111 percent of all ankle trauma ${ }^{(2)}$.

However, in discussing syndesmotic injury it seems the exact proximal and distal borders of the distal tibiofibular syndesmosis are not properly described.

There is no clear assertion in etiological ${ }^{(3)}$, genetic $^{(4)}$ or topographical ${ }^{(5)}$ fracture classifications, regarding the exact volume of the syndesmosis. Syndesmotic joint is now not in reality described in anatomical textual content books ${ }^{(6)}$ Kelikian $^{(7)}$ postulate that the distal tibiofibular joint starts origin at the level of the tibiofibular ligaments from the tibia and ends where those ligaments insert into the fibular malleolus.

\section{Materials and Methodology}

This is a prospective, time bound hospital based study performed in Rajah Muthiah Medical College, Chidambaram between July 2015 to September 2017. This study consist of 20 patients of syndesmotic disruption along with variable ankle fracture, who were evaluated preoperatively and intervened with appropriate fracture fixation and fixed with syndesmotic screws. All patients were informed about the study and informed written consent become received. All the patients were subsequently followed up for the period of 12 to 18 months. They were evaluated for the occurrence of ankle arthritis

\section{Inclusion criteria}

a) Patient who have been recognized to have distal tibiofibular syndesmotic disruption based on clinical and radiological strategies.

b) Age group among 18 and sixty five years.

c) Patient presented with less than 1 month old trauma.

d) Patient who have medical co -morbidities under control.

e) Patients who were medically fit for surgical procedure.

f) Patient who had been willing for the study and surgery.

\section{Exclusion criteria}

a) Patients less than 18 years and more than 65 years.

b) Patients who had greater than 1 month old trauma

c) Patients who have uncontrollable medical co-morbidities.

d) Patients who were medically not fit for surgery

e) Patients who had been not willing to undergo surgery or study.

\section{Data Collection and Analysis}

After the patient who have been identified with syndesmotic disruption was admitted after preliminary stabilisation with below knee slab. All the necessary medical information had been recorded within the profoma prepared for this study. All the cases with diagnosed syndesmotic disruption with fractures had been treated by means of syndesmotic screw $(4 \mathrm{~mm}$ cancellous screw) fixation. In doubtfull cases, to begin with the fractures have been fixed after which by means of performing cotton hook test, after confirming syndemotic disruption, it was fixed with $4 \mathrm{~mm}$ cancellous screws. Number of cortices to be purchased and number of screws needed was decided intra-operatively depending at the need of stability of disruption. Intra-operative datas recorded in the profoma. After completing the hospital treatment, patients were discharged and called for regular follow-up at 1,3,6,12 and 18 months. Meanwhile the need of screw removal will be decided at 3 months and dealt with 
therefore relying on the pain and restricted dorsiflexion and subsequently allowed for weight bearing.

All the patients have been clinically assessed by 3 months interval. Radiological assessment for progression and time of union, fracture alignment, implant related complications and occurrence of ankle arthritis have been analysed. Data collected at the end of the study was statistically analysed.

\section{Diagnostic Criteria for Syndesmotic} Disruption: As in all ankle injuries, clinical examination should be in a systematic approach. By palpation of the malleoli and other bony landmarks, consisting of the proximal fibula, fundamental ligamentous regions need to be assessed to exclude related issues. Many assessments were described. Apart from diagnostic check there are some standards which confirms syndesmotic disruption radiologically. They are

1. Fracture of posterior malleoli

2. Increase in medial clear apace

3. Disruption of ankle mortise

4. Anterior inferior tibio fibular ligament disruption proven with the aid of decrease in tibio fibular overlap

Other test are as follows: In the squeeze check, pain is elicited over the ankle joint as the distal tibia and fibula separate while the mid calf of the leg or just above is compressed. The fibular translation test, the Cotton test, and the crossover leg test have been additionally described ${ }^{(8)}$. In the fibular translation test, the examiner tries to move the fibula in the anterior-posterior plane: An increased translation, in comparison with the opposite side indicative of a syndesmosis injury.

In the Cotton hook test, the talus is moved in the mortise within the medial-lateral plane: Increased movement suggest a positive test. Crossed-leg test shows, the patient is seated with the injured leg over the normal knee; the involvement of the syndesmotic injury is identified when the pain is felt $^{(9)}$. A tibiofibular clear space more than $6 \mathrm{~mm}$ located $10 \mathrm{~mm}$ above the plafond is indicative of syndesmosis damage, while increase in space between the medial malleolus and the talus indicates syndesmotic and deltoid ligament disruption $^{(10)}$.

Finally all injured ankles were examined the use of intraoperative fluoroscopic external rotation stress assessments. The contralateral limb is used as a control. A external rotation force is carried out for stress examination. Stress test was repeated after both lateral and medial malleolar and syndesmotic fixation ${ }^{(11)}$.

\section{Management of the Patient}

As soon as the patient become admitted, a detailed history was taken and a meticulous examination of the patients was achieved. The informations were recorded in the profoma organized. X-ray ankle anteroposterior view, Mortise view and lateral view have been taken. Depending at the increase in medial clear space and reduced tibiofibular overlap, similarly the syndesmotic disruption become confirmed by means of stress dorsiflexion view. Further the fracture was classified according to Lauge Hansen classification. Only the fracture with syndesmotic disruption included in the study. Below knee slab applied and the limb was kept in Bohler braun splint until the patient became taken up for surgery. Patient was advised for non-weight bearing till the screw removal or upto 3 months. Subsequently allowed for weight bearing and followed up for 12-18 months and incidence of ankle arthritis was assessed.

\section{Long Term Follow-Up}

20 patients with syndesmotic disruption were fixed with $4 \mathrm{~mm}$ cancellous screws, and subsequently followed up in 3 months interval. Out of 20 patients 3 patients were complaining of persistent ankle pain. Among the three patients, screws were removed in two patients, one patient had screws insitu. These 3 patients along with other patients were followed up and progression of ankle arthritis were assessed. 3 patients had persistent ankle pain and arthritis developed in injured ankles. 


\section{JMSCR Vol||05||Issue||11||Page 30632-30639||November}

\section{Case-1}
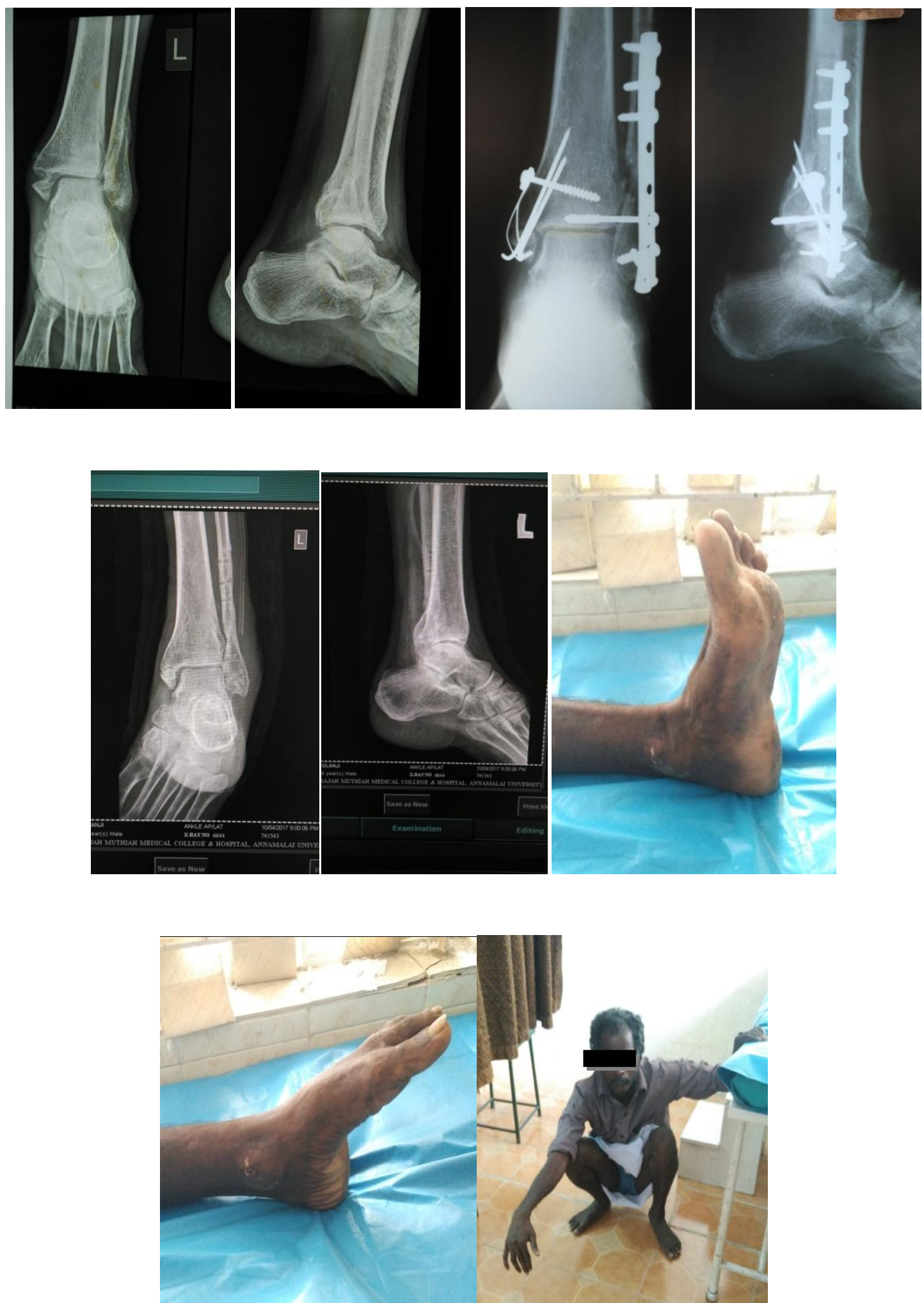


\section{JMSCR Vol||05||Issue||11||Page 30632-30639||November 2017}

\section{Case 2}
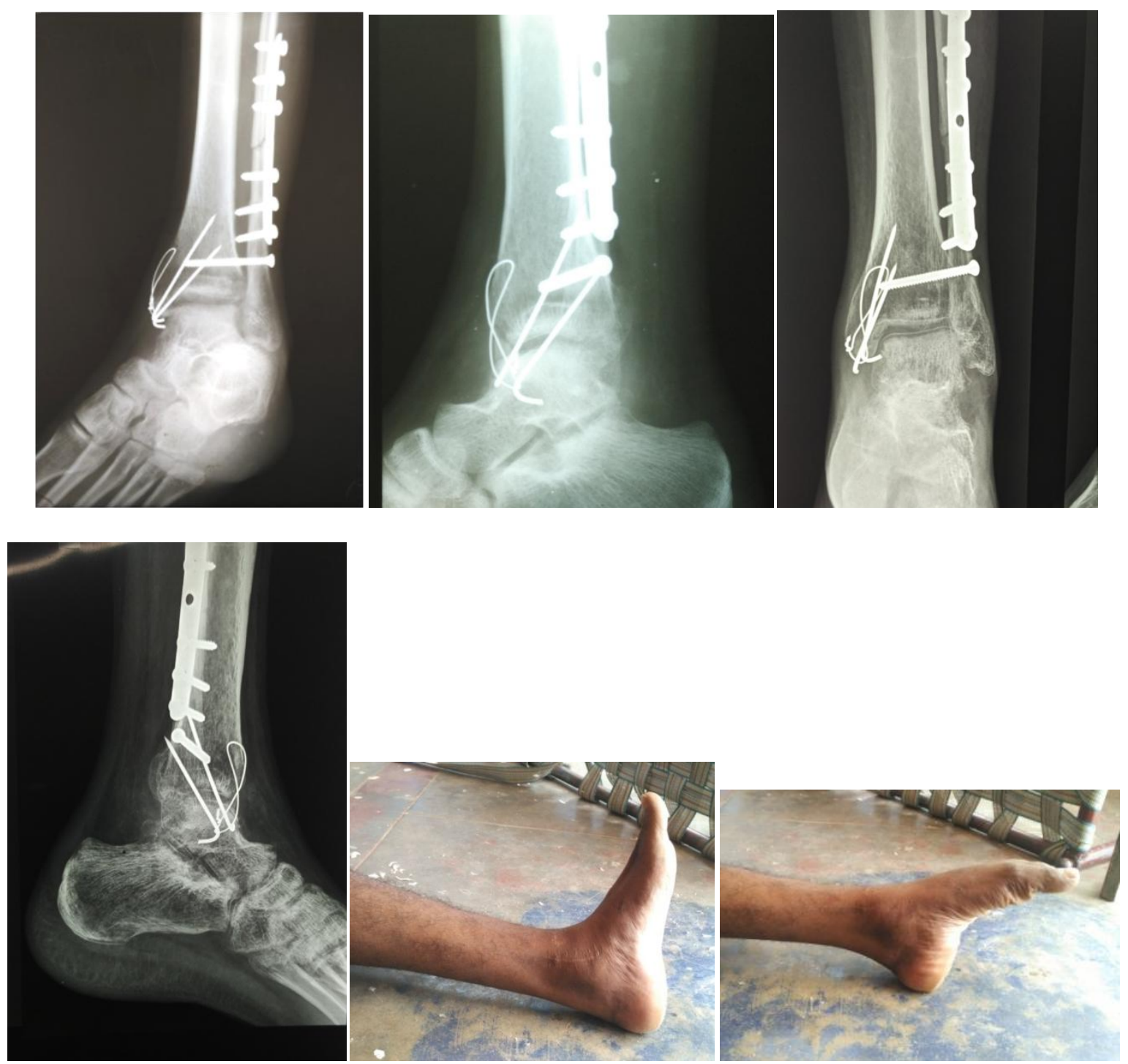

Case 3

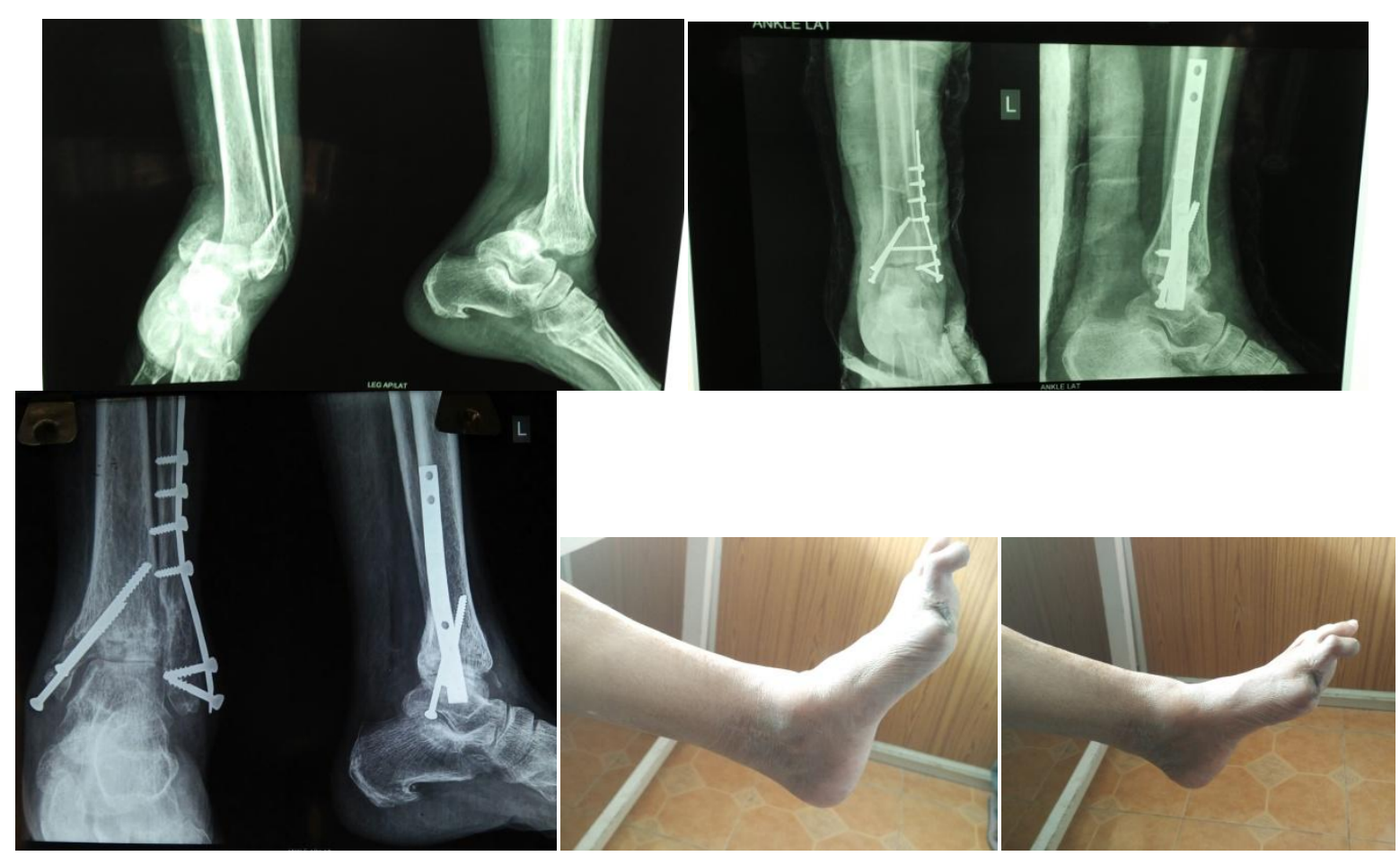


Table 1: Type of Fracture

\begin{tabular}{|l|c|}
\hline Type of fracture & $\begin{array}{c}\text { Number of } \\
\text { patients }\end{array}$ \\
\hline Trimalleolar fracture & 4 \\
\hline Bimalleolar fracture & 11 \\
\hline Only lateral malleolus fracture & 2 \\
\hline $\begin{array}{l}\text { Lateral malleolus fracture with } \\
\text { deltoid ligament disruption }\end{array}$ & 3 \\
\hline
\end{tabular}

Table 2: Complications

\begin{tabular}{|l|l|}
\hline \multicolumn{1}{|c|}{ Arthritis } & 3 \\
\hline Post operative infection & 1 \\
\hline Screw backout & 1 \\
\hline Late diastasis & 0 \\
\hline Tibio fibular synostosis & 0 \\
\hline
\end{tabular}

\section{Discussion}

Our study consists of 20 cases of closed ankle fractures with syndesmotic disruption diagnosed by radiological views and intra-operative tests. Maximum incidence of the injury was in the fifth decade of life. Injury was more common in males$12(60 \%)$ and females being 8(40\%). Right side was more commonly involved-12 patients (60\%).

Road traffic accidents contributed to $65 \%$ of injuries, followed by self fall while walking (35\%). Out of 20 patients, 14 are PER TYPE III pattern, 3 patients are PER TYPE IV pattern, 1 cases of PER TYPE II pattern and 2 cases of SER TYPE IV.

The most common injury pattern seen in our study was Pronation and external rotation type III. Stress radiographs are useful to assess ankle instability. Pronation external rotation injury type III and IV was very unstable and also associated syndesmotic disruption which should be diagnosed clinically and radiologically and must be fixed with syndesmotic screw from fibula to tibia, which may be stabilised by tricortical or quadricortical fixation depends on pattern of injury.

Functional outcome was equal for both tricortical and quadricortical fixation supported by Macleod mark et $\mathrm{al}^{(12)}$. But in fear of chance of screw breakage in quadricortical screw we usually prefer tricortical fixation. Usually we plan the whole implant removed at the end of 1 year. Till then the patients were allowed for weight bearing with screws insitu. David paul bell et al ${ }^{(13)}$ study shows maximum of three years of patient with retained screw without complications

In pronation external rotation injury fibular length restoration and rotation, ankle mortise and syndesmotic stability is important factor as noted by maverick et $\mathrm{al}^{(14)}$. We had good to excellent results in all cases as we could maintain the syndesmotic stability and fibular length by syndesmotic screws and fibular plating. Displacement is position of talus in the mortise and depends on uninjured deltoid ligament ${ }^{(15)}$.

Fixing only the malleolar fragment will not restore ankle stability in case of deltoid ligament rupture. It must be repaired subsequently if the deep deltoid ligament is torn ${ }^{(16)}$. Stable fractures shows no displacement in axial loading ${ }^{(17)}$. Treatment depends on the stability of fracture. Prognosis is depends on energy of injury. If the deltoid ligament is torn it must be repaired. Even though Lauge-Hansen classification describes in detail about the pattern of ankle fracture it does not deal with syndesmotic injuries According to Micheal Bekorom $^{(18)}$, pronation injuries/ type $\mathrm{C}$ weber fractures are usually associated with syndesmotic injuries than supination injuries/ type B weber injuries.

Miller study shows for ankle fractures syndesmotic injuries are fixed with screws and studied the functional outcome which was ranging from 75-85 of olerud and molander scoring system. ${ }^{(19)}$ Our study shows $20 \%$ of excellent result with score of 95 and 55\% shows good result with score of 8190, $25 \%$ shows fair results with some complications which were treated subsequently.

Among 20 patients, screws were removed around 12 weeks for 6 patients, and the whole implant including fibular plate and malleolar screw was removed with syndesmotic screws in 6 patients, 6 patients were on regular follow-up waiting for screw removal along with other implant, 2 patients were on long follow up who refuse for second surgery for implant removal was on regular followup without implant breakage but complaing of ankle pain. Further these patients were 
segregated and focused on ankle arthritis. On regular follow up there is progression of symptoms, were managed symptomatically and explained to the patients

On retrospective analysis, the reason for arthritis were found out. In one patient we advised delayed weight bearing as he managed initially by native bone setters in the form of oil bandages, later he was operated for syndesmotic disruption by screw fixation, but due to early weight bearing by the patient, he developed arthritic features. In second patient, he was a manual labourer, and advised delayed return to labour activities, as he had comminuted fibula fracture, but the patient did not followed up and later he ended up in arthritis. Third patient had diabetic mellitus with osteoporotic bone. She had spontaneous screw back out at the time of 10 weeks post operative period. Initially she had no arthritis, but had persistent ankle pain. But on long term follow up (after 1 year) she was ended in arthritis.

\section{Conclusion}

Though pronation and supination injuries produces syndesmotic disruption, we encounter most commonly the pronation-external rotation injury. In that PER TYPE III and IV are commonly seen. Though there are many controversies regarding need to fix syndesmotic disruption or not, our study concludes there is definitive need of fixation of syndesmotic disruption that too by syndesmotic screw to allow for early mobilisation, early return activities, to prevent ankle arthritis. Though there are various methods of fixation, in our study fixing with screws shows good functional outcome. Regarding the amount of cortical purchase we prefer tricortical purchase, which will avoid the implant breakage and helps in delayed implant removal as a whole, which helps to avoid multiple surgeries for implant removal.

Among the 20 patients, 3 patients had ankle arthritis which eventually started as chronic pain, then later on regular follow up ankle arthritis was noted both clinically and radiologically. Since the surgical fixation of syndesmotic injury was important to prevent ankle arthritis, three patients $(15 \%)$ of 20 patients had ankle arthritis, due to various avoidable factors.

\section{References}

1. Ramsey PL, Hamilton W. Changes in tibiotalar area of contact caused by lateral talar shift. J Bone Joint Surg Am. 1976;58:356-357

2. Hopkinson WJ, St Pierre P, Ryan JB, et al. Syndesmosis sprains of the ankle. Foot Ankle. 1990;10:325-330.

3. Ashhurst APC, Bromer RS. Classification and mechanism of fractures of the leg bones involving the ankle: based on a study of three hundred cases from Episcopal Hospital. Arch Surg. 1922;4:51-129.

4. Lauge-Hansen N. Fractures of the ankle. II. Combined experimental-surgical and experimental-roentgenologic investigations. Arch Surg. 1950;60:957985

5. Weber BG. Die Verletzungen des oberen Sprunggelenkes. Bern: Hans Huber; 1972. pp. 51-65

6. Lanz VT, Wachsmuth W. Praktische Anatomie. Berlin: Springer-Verlag; 1972. pp. 328-360

7. Kelikian H, Kelikian S. Disorders of the Ankle. Philadelphia: W.B. Saunders Company; 1985. pp. 4-8

8. Jenkinson, Richard J MD; Sanders, David W MD, MSc, FRCS(C); Macleod, Mark D MD, FRCS(C); Domonkos, Andrea BSc; Lydestadt, Jeanette RNJournal of Orthopaedic Trauma: October 2005 Volume 19 - Issue 9 - pp 604-609doi: 10.1097/01.bot.0000177114.13263.12

9. McCollum $\mathrm{G}$, van den Bekerom $\mathrm{M}$, Kerkhoffs G, Calder J, van Dijk C. Syndesmosis and deltoid ligament injuries in the athlete. Knee Surg Sports Traumatol Arthrosc. 2013; 21:1328-1337. doi: 10.1007/s00167-012-2205-1. 


\section{JMSCR Vol||05||Issue||11||Page 30632-30639||November}

10. Beumer A, Swiestra B, Moulders P. Clinical diagnosis of syndesmosis instability: evaluation of stress tests behind the curtains. Acta Orthop Scand. 2002; 73:667-669.

11. Cesar de Cesar P, Muller E. Comparison of MRI to physical examination for syndesmotic injuries after lateral ankle sprain. Foot Ankle Int. 2011;32:10-16. doi: 10. 3113/FAI. 2011.1110.

12. Manjoo, Ajay MD; Sanders, David W MD, MSc, FRCSC; Tieszer, Christina MSc; MacLeod, Mark D MD, FRCSC Journal of Orthopaedic Traum A doi: 10.1097/BOT.0b013e3181a9f7a5

13. David Paul Bell, Merng Koon Wong ${ }^{\mathrm{b}}$ Department of Orthopaedic Surgery, Changi General Hospital, 2 Simei Street 3, Singapore 529889, Republic of Singapore, Accepted 1 February 2006, Available online 19 April 2006

14. Yablon I.G., Segal .D., Leanch R.E., Ankle Injuries, New york, 1983, Churchill Livingstone.

15. Yablon I.G., Heller. F.G., Shouse .L; The Key role of lateral malleolus in displaced fractures of ankle: J. Bone Joint Surg : 59 A: 169, 1977. 